\title{
Gold Asymmetric-Split Ring Resonators (A-SRRs) for Proteins Sensing
}

\author{
Jharna Paul, Richard M. De La Rue and Nigel P. Johnson \\ Optoelectronics Research Group, School of Engineering, University of Glasgow, University \\ Avenue, G12 8LT, Glasgow, UK \\ Corresponding Authors: Jharna.Paul@Glasgow.ac.uk; Nigel.Johnson@Glasgow.ac.uk
}

\begin{abstract}
In this paper, gold asymmetric-split ring resonators (A-SRRs) are used for proteins sensing in the mid-infrared (IR) spectral region. Self-assembled monolayers (SAMs) of octadecanethiol (ODT) in ethanolic solution were deposited on the resonator surfaces to immobilise protein molecules for their detection. Different diameters ASRRs were fabricated on zinc selenide (ZnSe) substrates using electron-beam lithography technique. Their plasmonic responses appear in the mid-IR spectral region and match with the vibrational responses of many organic molecules. After the formation of SAMs layer, one sample was immersed in bovine serum albumin (BSA) solution for proteins adsorption while other sample was immersed in hydroxyl terminated hexa-ethylene glycol $(\mathrm{EG} 6-\mathrm{OH})$ solution to modify SAMs surfaces to resist immobilisation of proteins. The vibrational responses of these organic molecules, all samples were excited using an incident broadband mid-IR light source and their reflectance spectra were measured at normal incidence using a microscope coupled Fourier Transform Infrared (FTIR) spectrometer. This study highlights the capability of plasmonic structures (A-SRRs) fabricated on transparent and high refractive index ZnSe substrates allows the detection of BSA proteins with enhanced detection in the mid-IR spectral range, demonstrating their potential for a wide range of sensing applications, e.g. in biomedical engineering and food industries.
\end{abstract}

Keywords: Asymmetric spilt-ring resonators (A-SRRs), self-assembled monolayers (SAMs), octadecanethiol (ODT), phosphate buffer saline (PBS), hexa-ethylene glycol $\left(\mathrm{EG}_{6}-\mathrm{OH}\right)$ and bovine serum albumin (BSA)

\section{INTRODUCTION}

Advances in nanotechnology have facilitated the fabrication of biomedical-sensors and controlled the manipulation of bio-materials at nanoscale level, offering the opportunity to develop advanced sensing strategies in medical diagnosis. Improved diagnosis tools will increase life-expectancy and at the same time increasing the probability of people developing degenerative diseases, such as, Type-II diabetes and Alzheimer's. Proteins can denature and loses its activity upon changes in temperature, $\mathrm{pH}$, ion concentration and surface energy [1-3]. Proteins have an affinity to accumulate at the solid and liquid interface. Understanding the adsorption behavior of proteins on different surfaces and their specific detection plays a vibrant role for a wide range of applications, for example, in biomedical, biochemical engineering, food industries, and environmental monitoring [4-5]. Metallic-nanostructures can manipulate light - giving rise to localised and resonant enhancement of optical intensity - and hold potential for a wide-range of opticalsensing applications [6-8].

Bovine serum albumin (BSA) was chosen as a protein analyte because it is soluble in water and albumin is harmless so suitable for biomedical applications [2]. BSA is characterised by both prolate and oblate spheroid shape of dimensions $4 \times 4 \times 14 \mathrm{~nm}^{3}$. The cross-sectional area of BSA is $56 \mathrm{~nm}^{2}$ with molecular mass of $\sim 68$ $\mathrm{KDa}$. The secondary structure consists of components of mainly $\alpha$-helices along with a $\beta$-structure. The adsorption of BSA on different surfaces has been reported by several groups [9-11]. On hydrophobic surfaces, the adsorption of proteins is driven by the attraction of the nonpolar parts of the BSA molecules

Metamaterials X, edited by Allan D. Boardman, Nigel P. Johnson, Kevin F. MacDonald, Ekmel Özbay, Proc. of SPIE Vol. 9883, 98831C · @ 2016 SPIE · CCC code: 0277-786X/16/\$18 · doi: 10.1117/12.2230310 
towards the surface, whereas on a charged surface, the driving force is dominated by the electrostatic attraction between the surface and the oppositely charged functional groups alongside the polypeptide chain of the BSA molecules.

In this paper, the spectral response of $1 \mathrm{mg} / \mathrm{ml}$ adsorbed BSA proteins onto gold A-SRRs surfaces were measured using a microscope coupled to a Fourier Transform Infrared (FTIR) spectrometer. The plasmonic resonances of gold A-SRRs were tuned to match the vibrational responses of the proteins backgrounds in the mid-infrared (mid-IR) spectral region for their enhanced detection. The resonator surfaces were activated by forming self-assembled monolayers (SAMs) using $1 \mathrm{mM}$ octadecanethiol (ODT) in ethanol to attach proteins molecules for their detection. A related experiment was performed to prevent proteins molecules reaching the resonators surfaces. The microscopic images of plasmonic structures are also reported.

\section{MATERIALS AND METHODS}

\subsection{Materials}

Bovine Serum Albumin (BSA) - Lyophilized Powder with a molar mass of $68 \mathrm{kDa}, 1$-Octadecanethiol, purum, $\geq 95.0$ \% purity, Hexa (ethylene glycol) - 97\% Purity, Phosphate Buffered Saline (PBS) Powder@ pH: 7.4; all materials were purchased from the Sigma-Aldrich Company, U.K. The proteins were used as received.

\subsection{Asymmetric-Split Ring Resonators (A-SRRs)}

Four different diameters $1.0-1.15 \mu \mathrm{m}$ of asymmetric-split ring resonators (A-SRRs) with an incremental step of $50 \mathrm{~nm}$ of were fabricated on zinc selenide $(\mathrm{ZnSe})$ substrates using the electron-beam lithography technique, followed by gold-metallisation and subsequent lift-off. The detailed characterisation of these ASRRs using both the simulation and fabrication techniques were described elsewhere by the authors [12]. The reflectance spectra of the A-SRRs were measured at normal incidence, with the incident electric field (Efield) vector polarised in the Y-direction, as shown in Fig. 1(a), using a microscope-coupled FTIR spectrometer. A broadband mid-IR source was used to excite the sample - and the beam was polarised using a ZnSe IR polariser. An iris was used to restrict the beam size to an area of $\sim 50 \times 50 \mu \mathrm{m}^{2}$. The spectra were normalised against plane gold to obtain the responses of the A-SRRs alone. The results are shown in Fig. 1 (b), where the positions of both resonances were monotonically red-shifted to the longer wavelengths side of the spectrum as the ring diameter increased The scanning electron micrograph (SEM) image of an array of fabricated A-SRRs are shown in Fig. 1 (c).

(a)

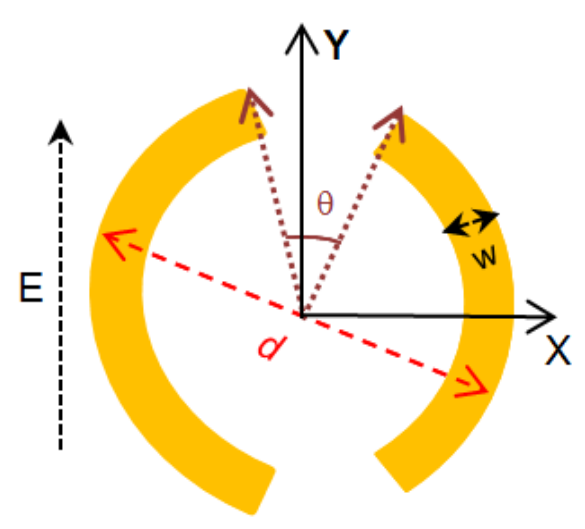

(b)

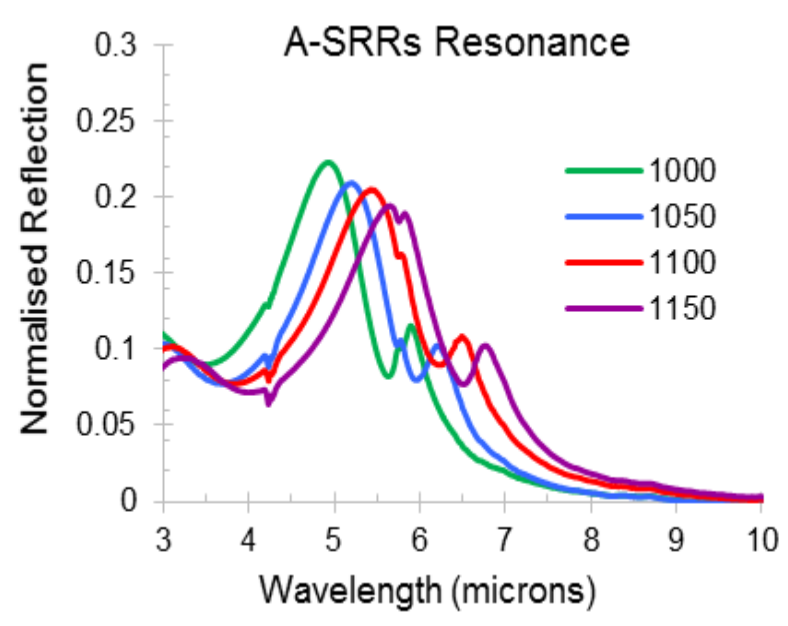


(c)

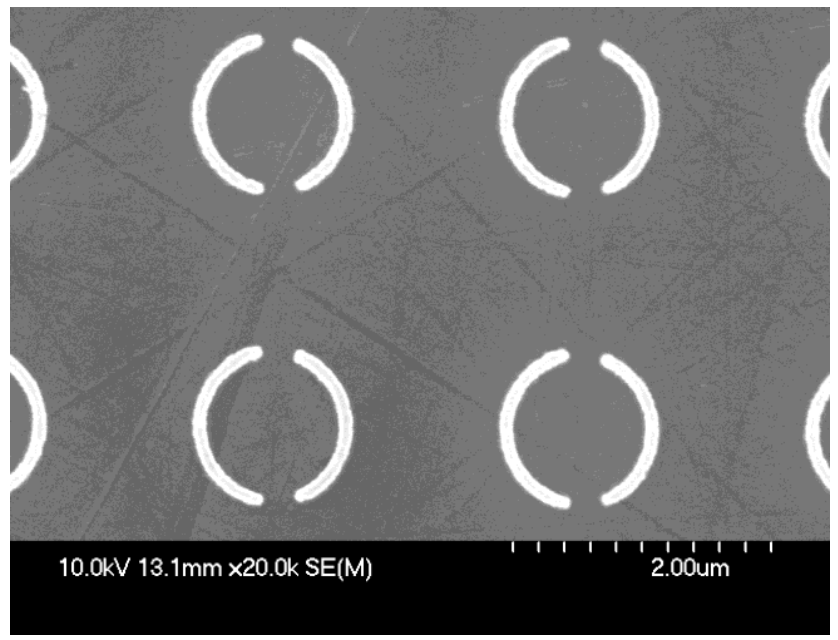

Fig. 1 (a) Schematic of an A-SRR with diameter $d$; gap angle, $\theta=30^{\circ}$ and strip width, $w=100 \mathrm{~nm}$. (b) Normalised reflectance response of A-SRRs with diameters 1.0, 1.05, 1.1 and $1.15 \mu \mathrm{m}$ respectively. (c) Scanning electron micrograph (SEM) image of an array of A-SRRs.

\subsection{Self-Assembled Monolayers (SAMs)}

The formation of self-assembled monolayers (SAMs) were carried out by immersing three identical gold ASRRs with four different diameters in $1 \mathrm{mM}$ 1-octadecanethiol $\left(\mathrm{CH}_{3}\left(\mathrm{CH}_{2}\right)_{17} \mathrm{SH}\right)$ diluted in ethanolic solution for $\sim 24$ hours at ambient temperature. After this, the samples were rinsed with pure ethanol twice to remove physio-absorbed multilayers and then blown dry using nitrogen. The longer immersion time was chosen to form well-ordered assemblies and also to simplify the assembly procedures without requiring surface modifications before their immersion [13-14].

\subsection{Immobilisation of Proteins}

Immediately after the formation of SAMs on resonator surfaces, the sample was immersed in $1 \mathrm{mg} / \mathrm{ml}$ of bovine serum albumin (BSA) solution in phosphate buffer saline (PBS) at $\mathrm{pH} 7.4$ for 2 hours at room temperature. After this, the sample was washed twice using the distilled, de-ionised RO water and then blown dry with nitrogen.

A second experiment was performed to resist proteins molecules reaching the resonator surfaces. For this, the sample was immersed in $1 \mathrm{mM}$ hydroxyl-terminated hexa-ethylene glycol $\left(\mathrm{EG}_{6}-\mathrm{OH}\right)$ solution to modify the SAMs surfaces formed earlier on top of gold A-SRRs, and then subsequently immersed in the same concentration of BSA solutions described above.

\section{SENSOR CHARACTERISATION}

\subsection{Detection of Octadecanethiol}

To detect the molecular responses of $1 \mathrm{mM}$ octadecanethiol (ODT), the reflectance spectra were measured at normal incidence using the FTIR spectrometer over an area of $50 \times 50 \mu \mathrm{m}^{2}$ and the results are shown in Fig. 2 (a) where the signal enhancement ( 6\%) due to the adsorption of the thiol molecules (red colour) compared to that of the A-SRRs responses alone (blue colour). Fig. 2 (b) shows the magnified spectra over the region $3.3-3.6 \mu \mathrm{m}$ showing both the asymmetric and symmetric $\mathrm{CH}_{2}$ stretching vibrations of the ODT molecules at 
$3.42 \mu \mathrm{m}$ and $3.5 \mu \mathrm{m}$ respectively through the intensity dips of the reflection spectrum. The intensity difference between the minimum dips and the adjacent maximum peaks of the modes are calculated to be $0.25 \%$ and $0.14 \%$ respectively.

(a)

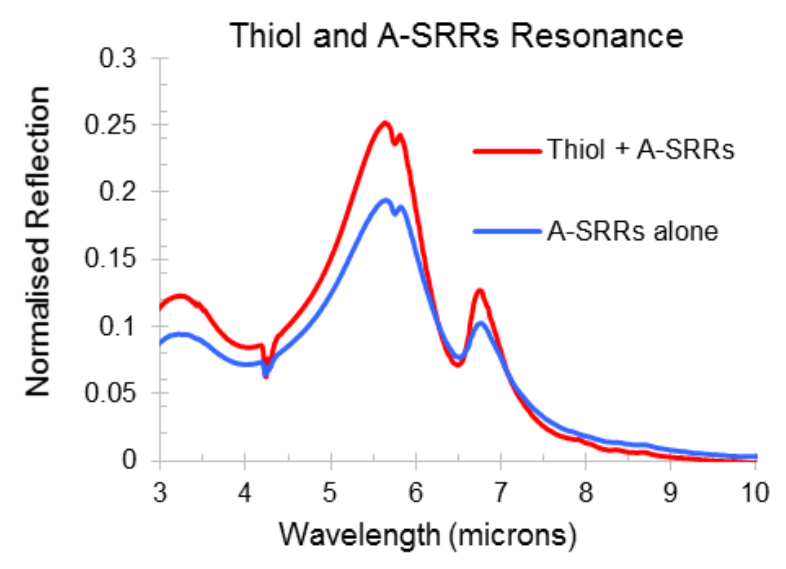

(b)

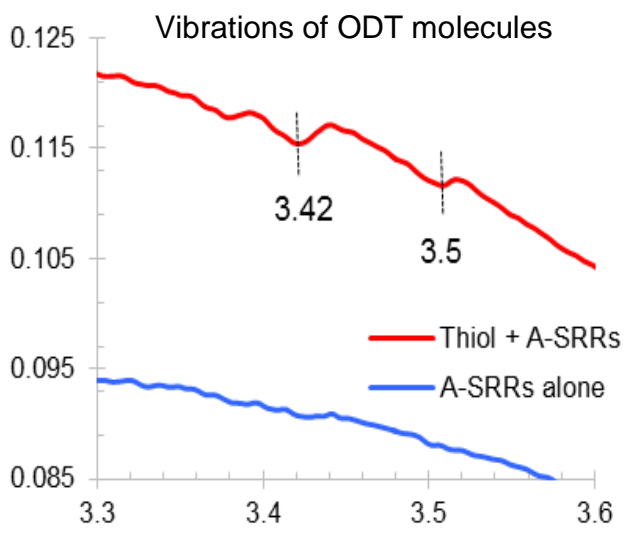

Fig. 2(a) Comparison of the spectral response of the $1 \mathrm{mM}$ ODT adsorbed on gold A-SRRs surfaces and without thiol (A-SRRs alone). (b) the magnified spectra showing the molecular resonaces of ODT.

Bain et al. [13] have reported the measured thickness of the monolayer of $1 \mathrm{mM}$ ODT on gold to be $20 \pm 2 \AA$ but for this work, the atomic force microscopy (AFM) measurements show the formation of multilayer assemblies for the same $1 \mathrm{mM}$ concentration thiol used, which is a matter of further investigation.

\subsection{Detection of Proteins}

Fig. 3(a) shows the adsorption spectra of the $1 \mathrm{mg} / \mathrm{ml}$ BSA proteins for an incubation time of 2 hours, along with the magnified spectra of the amide I and II bands are shown in Fig. 3 (b) over the wavelength range 5.9 $6.7 \mu \mathrm{m}$. The amide I band is centered at $\sim 5.88-6.25 \mu \mathrm{m}$ is mainly due to $\mathrm{C}=\mathrm{O}$ stretching vibrations [10-11 and references there in]. For amide I component bands, it is clearly showing the random coils/chains at $\sim 6.08$ $\mu \mathrm{m}$, with the development in free external $\alpha$-helices or extended chains at $\sim 6.1 \mu \mathrm{m}$, along with the extended chains $/ \beta$-sheets at $\sim 6.18 \mu \mathrm{m}$ and intermolecular bonding at $\sim 6.28 \mu \mathrm{m}$. Similarly, the amide II band centred at $~ 6.25-6.7 \mu \mathrm{m}$ consists of the $\mathrm{NH}$ bending and $\mathrm{CN}$ stretching vibrations. Similarly, the formation of the amide II band at $\sim 6.5 \mu \mathrm{m}$ is clearly visible.

(a)

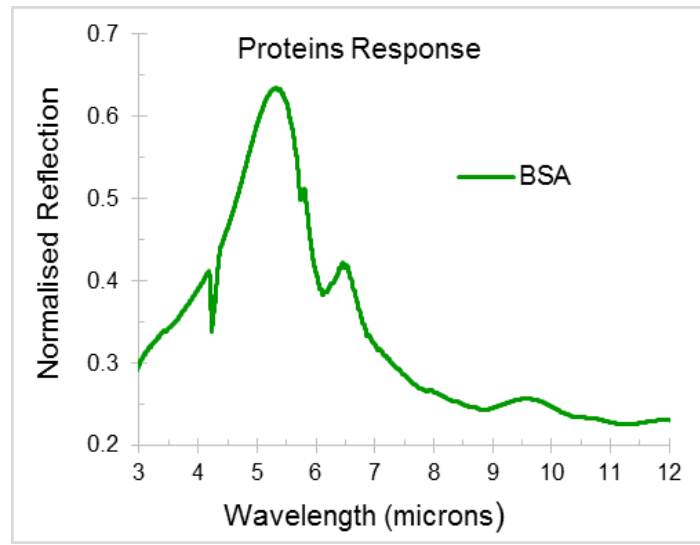

(b)

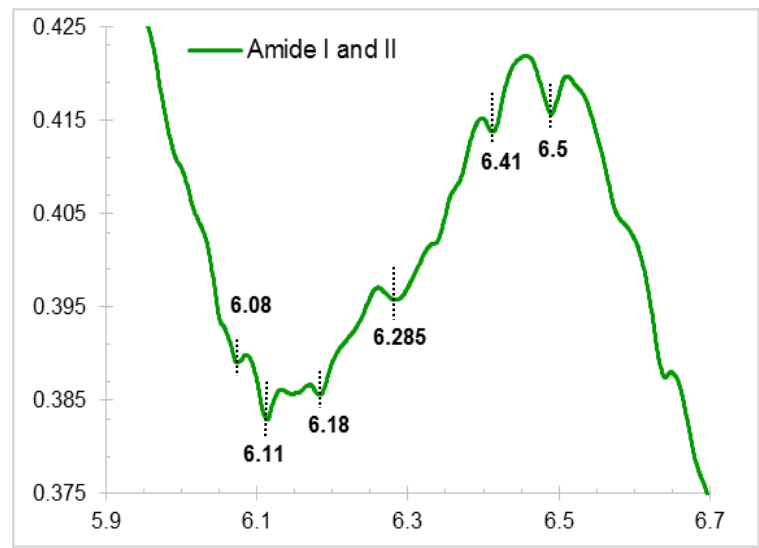

Fig. 3 (a) The normalised reflectance response of the $1 \mathrm{mg} / \mathrm{ml}$ BSA Proteins spectra over an area of $300 \mathrm{x}$ $300 \mu \mathrm{m}^{2}$. (b) magnified spectra showing the amide I and II vibrational responses from $5.9-6.7 \mu \mathrm{m}$. 


\subsection{Hexaethylene Glycol ( $\left.\mathrm{EG}_{6}-\mathrm{OH}\right)$ to Resist Adsorption of Proteins}

The oligo/poly ethylene glycol (EG)-terminated self-assembled monolayers (SAMs) on gold surfaces were found to be highly resistant to the adsorption of proteins [15-16]. The experimental results further confirm that the hydroxyl terminated hexa-ethylene glycol $\left(\mathrm{EG}_{6}-\mathrm{OH}\right)$ functionalised SAMs on gold A-SRRs surfaces prevent the adsorption of proteins due to the interfacial water adsorption on the ethylene moieties in the SAMs, and are shown in Fig. 4 (a) where Fig. (b) shows the magnified spectra over the range $5.9-6.7 \mu \mathrm{m}$ with no vibrational responses of proteins backgrounds. Fig. 4 (c) clearly shows the molecular vibrational adsorptions of $\mathrm{EG}_{6}-\mathrm{OH}$ due to the $\mathrm{C}-\mathrm{O}-\mathrm{C}$ stretching vibrations at $\sim 8.89 \mu \mathrm{m}$ along with the ether $\mathrm{CH}_{2}$ twisting and rocking modes at $\sim 7.8$ and $10.52 \mu \mathrm{m}$ respectively.

(a)

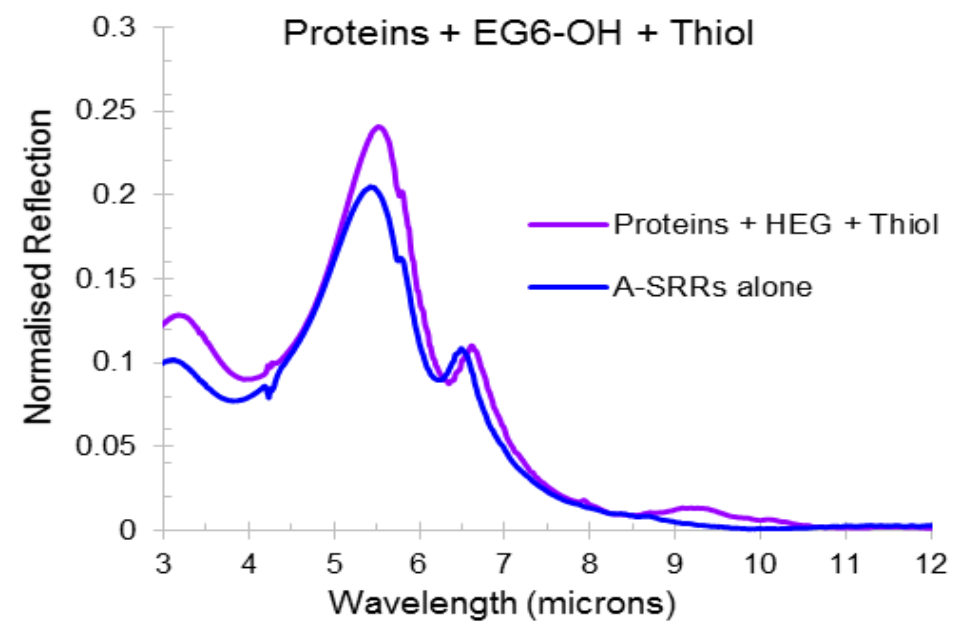

(b)

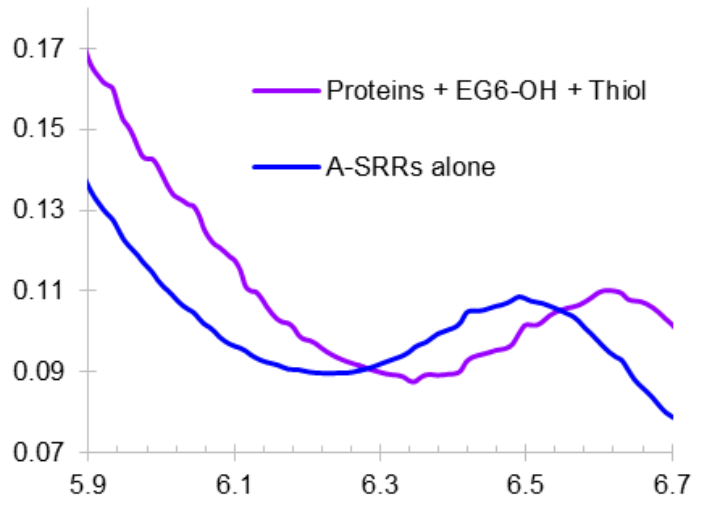

(c)

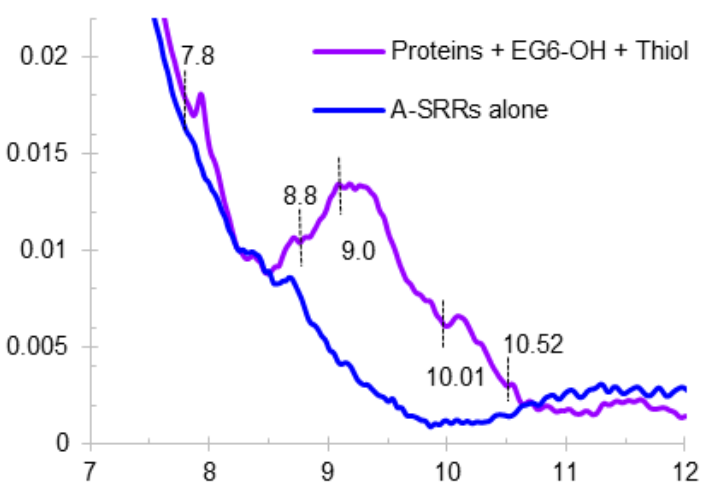

Fig. 4 (a) The normalised reflectance spectra of $\mathrm{EG}_{6}-\mathrm{OH}$ functionalised SAMs on gold A-SRRs surfaces. (b) the magnified spectra showing no adsorption of BSA proteins. (c) the vibrational adsorption bands of the $\mathrm{EG}_{6}-\mathrm{OH}$ unit. 


\section{DISCUSSION AND CONCLUSION}

The adsorption of $1 \mathrm{mg} / \mathrm{ml} \mathrm{BSA}$ proteins on gold asymmetric-split ring resonators (A-SRRs) surfaces were experimentally detected. The SAMs were spontaneously formed by immersing the samples into the $1 \mathrm{mM}$ octadecanethiol (ODT) in ethanol solution. The vibrational responses of the ODT molecules are clearly visible through dips in the reflection spectrum measured using the FTIR spectrometer. Although the plasmonic responses of gold A-SRRs did not match well with the vibrational backgrounds of ODT molecules, still their presence are clearly detectable. However by matching the plasmonic responses with the molecular vibrations, signal enhancement could be further improved - which is an ongoing target. For adsorption of $1 \mathrm{mg} / \mathrm{ml} \mathrm{BSA}$ proteins, the development in free external $\alpha$-helices or extended chains at $\sim 6.1 \mu \mathrm{m}$ along with the extended $\beta$-sheets and intermolecular bonding are identified using the FTIR spectra. Also modification of SAMs surfaces shows their ability to resist the adsorption of proteins into the pre-activated gold A-SRRs.

In summary, different diameters gold A-SRRs were fabricated on zinc selenide ( $\mathrm{ZnSe}$ ) substrates using the electron-beam lithography and have successfully modified their surfaces to immobilise proteins molecules for their detection. Also modification of SAMs surfaces using hydroxyl-terminated hexa-ethylene glycol $\left(\mathrm{EG}_{6}-\mathrm{OH}\right)$ demonstrates their ability to resist proteins reaching the gold resonators surfaces, very promising for the proteins blocking applications. The plasmonic structures (A-SRRs) inscribed on transparent and high refractive index $\mathrm{ZnSe}$ substrates shows their ability to detect the vibrational responses of organic molecules, particularly proteins with enhanced performance holds potential for a wide range of biomedical applications in the mid-IR spectral range.

\section{ACKNOWLEDGEMENT}

Jharna Paul acknowledges the Daphne Jackson Trust (DJT) for providing her with a Fellowship to overcome a career gap and the James Watt Nanofabrication Centre (JWNC) at the University of Glasgow for the fabrication facilities. She also thanks Thomas Reilly for his additional technical support.

\section{REFERENCES}

[1] McClellan, S. J. and Franses. E. I., "Effect of concentration and denaturation on adsorption and surface tension of bovine serum albumin," Colloids Surf. B: Biointerfaces, 28, 63-75 (2003).

[2] Murayama, K. and Tomida, M., "Heat-induced secondary structure and conformation change of bovine serum albumin investigated by Fourier Transform Infrared Spectroscopy," Biochemistry, 43. 11526-11532 (2004).

[3] Kopac, T., Bozgeyik, K. and Yener, J., "Effect of $\mathrm{pH}$ and temperature on the adsorption of bovine serum albumin onto titanium dioxide," Coll. and Surf. A: Phystcochem. Eng. Aspects, 322, 19-28 (2008).

[4] Ziegler, G. R. and Foegeding E. A., "The Gelation of Proteins," Adv. Food Nutr. Res. 34, 203-298 (1990).

[5] Nakanishi, N., Sakiyama, T. and Imamura. K. "On the adsorption of proteins on solid surfaces, a common but very complicated phenomenon," J. of Bioscience and Bioengineering. 91 (3), 233-244 (2001) DOI: 10.1016/s1389-1723(01) 80127-4.

[6] Lahiri, B, Khokhar, A. Z., De La Rue, R. M., McMeekin, S. C. and Johnson, N. P., "Asymmetric split ring resonators for optical sensing of organic materials," Opt. Exp., 17(2), 1107-1115 (2009).

[7] Neubrech, F., Pucci, A., Cornelius, T. W., Karim, S., -Etxarri, A. G. and Aizpurua, J., "Resonant plasmonic and vibrational coupling in a tailored nanoantenna for infrared detection," Phys. Rev. Lett., 101, 157403-1-4 (2008).

[8] Mbomson, I. G., McMeekin, S., Lahiri, B., De La Rue, R. M. and Johnson, N. P., "Gold asymmetric split ring resonators for nano sensing of estradiol," Proc. Of SPIE, 9125, 9125O-1 (2014) DOI: 10.1117/12.2052578. 
[9] Fitzpatrick, H., Luckham, P. F., Eriksen, S. and Hammond, K., "Bovine serum albumin adsorption to mica surfaces," Coll. and Surf. 65, 43-49 (1992).

[10]Roach, P., Farrar, D. and Perry, C. C., "Surface tailoring for controlled protein adsorption: effect of topography at the nanometer scale and chemistry," J. Am. Chem. Soc. 128, 3939-3945 (2006).

[11] Jeyachandran, Y. L., Mielczarski, E., Rai, B. and Mielczarski, J. A., "Quantitative and qualitative evaluation of adsorption/desorption of bovine serum albumin on hydrophilic and hydrophobic surfaces," J. Am. Chem. Soc. 25 (19), 11614-11620 (2009).

[12] Paul, J., Sharp, G. J., Lahiri, B., De La Rue, R. M. and Johnson, N. P., "Simulation and experimental responses of the asymmetric-split ring resonators (A-SRRs) for sensing applications in the midinfrared region," $9^{\text {th }}$ Int. Congress on Adv . Electromag. Mat. in Microwaves and Optics Metamaterials 2015, 1029-31 (2015), ISBN 978-88-941141-0-2.

[13] Bain, C. D., Troughton, E. B., Tao, Yu-T., Evall, J., Whitesides, G. M. and Nuzzo, R. G., "Formation of monolayer films by the spontaneous assembly of organic thiols from solution onto gold," J. Am. Chem. Soc. 111 (1), 321-335 (1989).

[14] Ishida, T., Tsuneda, S., Nishida, N., Hara, M., Sasabe H. and Knoll. W., "Surface-conditioning effect of gold substrates on octadecanethiol self-assembled monolayer growth," Langmuir. 13, 4638-4643 (1997).

[15] Harder, P., Grunze, M., Dahint, R., Whitesides, G. M. and Laibinis, P. E., "Molecular conformation in oligo (ethylene glycol)-terminated self-assembled monolayers on gold and silver surfaces determines their ability to resist protein adsorption," J. Phys. Chem. B, 102, 426-436 (1998).

[16] Wang, R. L. C., Kreuzer, H. J. and Grunze, M., "Molecular conformation and solvation of oligo (ethylene glycol)-terminated self-assembled monolayers and their resistance to protein adsorption," J. Phys. Chem. B. 101, 9767-9773 (1997). 